\title{
Assessment of Cerebrospinal Fluid Hydrodynamics Using Magnetic Resonance Imaging in Postcraniospinal Surgery Patients
}

\author{
Pankaj Arora ${ }^{1}$ Kanica Rawat ${ }^{2}$ Rajiv Azad ${ }^{2}$ Kehkashan Chouhan ${ }^{2}$ \\ ${ }^{1}$ Department of Neurosurgery, Shri Guru Ram Rai Institute of \\ Medical and Health Sciences, Patel Nagar, Dehradun, Uttarakhand, \\ India \\ 2Department of Radiodiagnosis, Shri Guru Ram Rai Institute of \\ Address for correspondence Rajiv Azad, Professor and Head, \\ Department of Radiodiagnosis, Shri Guru Ram Rai Institute of \\ Medical and Health Sciences, Patel Nagar, D6, Medical Campus, \\ Dehradun, Uttarakhand, India (e-mail: rajivas23@yahoo.com).
} Medical and Health Sciences, Patel Nagar, Dehradun, Uttarakhand, India

Indian J Radiol Imaging :2021;31:304-310

\begin{abstract}
Keywords

- cerebrospinal fluid

- craniospinal

- PCMRI

Objective Aim of this study is to evaluate the effect of craniospinal interventions on cerebrospinal fluid (CSF) flow hydrodynamics and study the correlation of postoperative changes in flow alteration with clinical outcome.

Materials and Methods Fifty patients who underwent various craniospinal procedures were studied using conventional and phase-contrast magnetic resonance imaging (PCMRI) protocol. CSF flow quantification was performed at cerebral aqueduct, foramen magnum, C2-3, and D12-L1 vertebral levels with site showing maximal alteration of CSF flow dynamics considered as the region of interest. Velocity encoding was kept at $20 \mathrm{~cm} / \mathrm{s}$. Patients with pathology atcraniovertebral junction were considered separately (group I) from others (group II) due to different flow dynamics. Follow-up scans were performed after an interval of 1 month for temporal evaluation of changes in CSF flow dynamics.

Results Patients in both groups showed a significant change in peak CSF velocity postoperatively (mean change of $1.34 \mathrm{~cm} / \mathrm{s}$ in group I and $0.28 \mathrm{~cm} / \mathrm{s}$ in group II) with bidirectional improvement in flow on cine-phase-contrast qualitative images. Regional pain $(82 \%)$ and headache (46\%) were seen in most of the patients preoperatively. Postoperatively clinical symptoms improved in $59.5 \%$, static in $26.2 \%$, and worsened in $14.3 \%$. In both the groups, an improvement in clinical symptomatology had significant correlation with mean changes in peak CSF velocity postoperatively ( $p=0.04$ in both groups).

Conclusion PCMRI can effectively evaluate changes in CSF flow noninvasively both pre- and postoperatively. This may have potential role in determining clinical outcome and prognosis of patients undergoing procedures in craniospinal axis.
\end{abstract}

published online July 27,2021
DOI https://doi.org/

$10.1055 / \mathrm{s}-0041-1734362$ ISSN 0971-3026
(C) 2021. Indian Radiological Association.

This is an open access article published by Thieme under the terms of the Creative Commons Attribution-NonDerivative-NonCommercial-License, permitting copying and reproduction so long as the original work is given appropriate credit. Contents may not be used for commercial purposes, or adapted, remixed, transformed or built upon. (https://creativecommons.org/licenses/by-nc-nd/4.0/).

Thieme Medical and Scientific Publishers Private Ltd. A-12, Second Floor, Sector -2, NOIDA -201301, India 


\section{Introduction}

Cerebrospinal fluid (CSF) shows complex oscillatory flow during the cardiac cycle across different levels with areas of relative change in velocity and flow patterns. ${ }^{1}$ CSF flow may be obstructed to a varying degree in different pathologies leading to a wide array of clinical and neuroradiological presentations requiring various surgical interventions. ${ }^{2}$ It is imperative to develop noninvasive predictors of surgical outcome by imaging so as to prognosticate about the potential benefits. Ongoing advancements in magnetic resonance imaging (MRI) and development of phase-contrast MRI (PCMRI) have allowed a qualitative and quantitative assessment of CSF flow which is accurate and reproducible.

There is only limited literature available outlining the clinical applications of PCMRI in determining the changes of CSF flow in postsurgical scenario with no recognized literature on temporal evaluation in such patients. ${ }^{3,4}$ The present study evaluated the alteration of the CSF flow hydrodynamics postsurgically and its correlation with clinical outcome.

\section{Materials and Methods}

The study was a longitudinal study with a case-control design comprising 50 patients and 25 controls, conducted over a period of 2 years in a tertiary-care hospital in India. Patients with various craniospinal pathologies causing CSF flow alterations undergoing surgeries irrespective of age and gender comprised the study group. Prior approval of the Institutional Ethical Committee (IEC approval date: May 28, 2018) was taken. Healthy subjects formed the control group.

\section{Data Collection Techniques and Tools}

Conventional magnetic resonance imaging of the brain was performed before PCMRI. For qualitative PCMRI, a two-dimensional fast low-angle shot (FLASH) sequence was used. T2-weighted sagittal images, phase, rephase, and magnitude images were acquired. Imaging parameters were repetition time/echo time $(\mathrm{TR} / \mathrm{TE})=34.45 / 9.73 \mathrm{~ms}$, field of view $($ FOV $)=240 \mathrm{~mm}$, flip angle $=10$ degrees, and slice thickness $=4.5 \mathrm{~mm}$. CSF flow dynamics were quantitatively studied by using a prospective cardiac-gated (pulse trigger being used for cardiac synchronization) high-resolution axial phase-contrast protocol with an optimal imaging plane depending on surgical site perpendicular to (1) mid one-third of the cerebral aqueduct, (2) foramen magnum, (3) C2-3, and (4) D12-L1 vertebral levels. The imaging parameters were $\mathrm{TR} / \mathrm{TE}=41.35 / 4.09 \mathrm{~ms}, \mathrm{FOV}=320 \mathrm{~mm}$, flip observer was taken angle $=30$ degrees, and slice thickness $=5 \mathrm{~mm}$. Velocity encoding was kept at $20 \mathrm{~cm} / \mathrm{s}$. Area with maximal alteration of cerebrospinal flow dynamics in patients was selected as the region of interest (ROI) for comparison.

The acquired FLASH through-plane images were transferred to Argus Siemens postprocessing program. In all cases, CSF flow was initially evaluated qualitatively following acquisitions of phase, rephase, and magnitude images. A circular ROI was placed carefully on each image manually in one of those series. In all controls and patients, the following parameters were assessed at different levels as follows: (1) area of subarachnoid spaces (SAS), (2) peak velocity, (3) average velocity, (4) forward volume, (5) reverse volume, and (6) stroke volume. Two radiologists, who were blinded to the clinical history, evaluated the images qualitatively and quantitatively. The average values of quantitative parameters calculated by the observers were taken.

Assessment of clinical signs and symptoms was done preand postoperatively. Based on clinical examination, patients were categorized into the following groups improved, unchanged, and deteriorated accordingly. Each presenting symptom was scored preoperatively and postoperatively between 1 and 5, with 1 representing no disability and 5 representing complete disability. Complete disability was considered when patients had significant deterioration in activities of daily living or inability to carry out his/her routine occupational job. Cumulative accrued values based on each symptom were averaged. Postoperative assessment in patients was performed 1 month after the surgical intervention, subject to patient availability and informed consent.

\section{Statistical Analysis}

Statistical description of data was done in terms of mean ( \pm standard deviation), frequencies (number of cases), and percentages. Paired $t$-test was used to compare the mean change in peak CSF systolic velocity pre- and postoperatively. Changes in clinical grading and symptoms were evaluated using Kendal's tau test. The Mann-Whitney $U$-test and Kruskal-Wallis tests were used to compare the mean change. The $p$-value of $<0.05$ was considered significant. All the analysis was performed on SPSS 16.0 version (Chicago, Inc., United States).

\section{Results}

Fifty patients and 25 controls were included in the study. The mean age of patients was $42.36 \pm 21.38$ and of controls was $38.56 \pm 18.69$ years.

The primary presenting symptoms in study participants were regional pain (82\%) and headache (46\%). Other symptoms included limb weakness (24\%), visual disturbance (12\%), and gait disturbance (8\%). Postoperative imaging follow-up

Table 1 Cumulative disability scoring of clinical symptoms

\begin{tabular}{|l|l|l|l|}
\hline Group & Number of patients & Preoperative disability score & Postoperative disability score \\
\hline Improved & 25 & 15.88 & 9.6 \\
\hline Static & 11 & 14.04 & 13.04 \\
\hline Worsened & 6 & 14.9 & 15.7 \\
\hline
\end{tabular}


was done in 44 patients after an interval of 1 month. Two infants did have postsurgical follow-up. However, accurate assessment of clinical symptomatology improvement, especially pain, was not achieved. Postoperatively, a majority (59.5\%) of the patients showed improvement in clinical symptoms. Eleven patients (26.2\%) showed no change, while six patients (14.3\%) showed deterioration. A greater disability score reduction favored an improved clinical outcome (-Table 1).

\section{Phase-Contrast Magnetic Resonance Imaging}

Quantitative parameters measured at cerebral aqueduct, foramen magnum, C2-3, and D12-L1 vertebral levels, in controls, showed no significant difference in peak CSF velocities. An overview of the calculated parameters of CSF peak velocity is compiled in - Table 2. Qualitative analysis on cine MR images showed normodynamic pulsatile bidirectional flow with typical sinusoidal waveforms. However, in patients, a strikingly high variability of flow pattern and quantitative values was observed, particularly at ROI, with an irregular low-amplitude nonpulsatile waveform at site of alteration in most of the patients. The highest peak CSF velocity was noted in cervical SAS ventrally in both patients and controls, that is, 3.04 and $3.25 \mathrm{~cm} / \mathrm{s}$, respectively.

At ROI, an overall higher peak CSF velocity was obtained postoperatively (postoperatively: $2.51 \mathrm{~cm} / \mathrm{s}$ vs. preoperatively: $2.16 \mathrm{~cm} / \mathrm{s}$ ). However, the comparative difference was insignificant owing to large range of altered flow parameters.

Table 2 Mean cerebrospinal fluid peak velocity in controls and patients

\begin{tabular}{|l|l|l|}
\hline \multirow{2}{*}{ Region of interest } & \multicolumn{2}{|c|}{ Peak systolic velocity $(\mathrm{cm} / \mathbf{s})$} \\
\cline { 2 - 3 } & Control & $\begin{array}{l}\text { Patient } \\
\text { (preoperative) }\end{array}$ \\
\hline Aqueduct & 2.14 & 2.28 \\
\hline $\begin{array}{l}\text { Foramen magnum } \\
\text { (ventral) }\end{array}$ & 2.74 & 2.14 \\
\hline Foramen magnum (dorsal) & 2.80 & 2.74 \\
\hline C2-3 (ventral) & 2.32 & 2.80 \\
\hline C2-3 (dorsal) & 2.10 & 2.32 \\
\hline D12-L1 (ventral) & 3.04 & 2.10 \\
\hline D12-L1 (dorsal) & 3.25 & 3.04 \\
\hline
\end{tabular}

Patients were divided into two subgroups due to differences in pattern of flow alteration, that is, group I: ROI at craniocervical junction and group II: ROI at aqueduct, cervical, and dorsolumbar canal. Groups I and II both showed improvement in CSF flow at ROI on dynamic cine MR images in most of the patients. However, a varied response of change in CSF peak velocity was noted to surgical intervention. Group I showed an overall reduction in peak CSF velocity postoperatively (mean reduction of $\sim 1.34 \mathrm{~cm} / \mathrm{s}$ ), and in group II, an overall increase (mean increase of $\sim 0.28 \mathrm{~cm} / \mathrm{s}$ ) was observed.

Comparison of quantitative values in patients pre- and postoperatively at ROI showed significant differences in values of peak CSF velocity in both groups I and II which showed a significant correlation ( $p=0.04$ in both) with improvement in clinical outcome (improved versus others) as illustrated
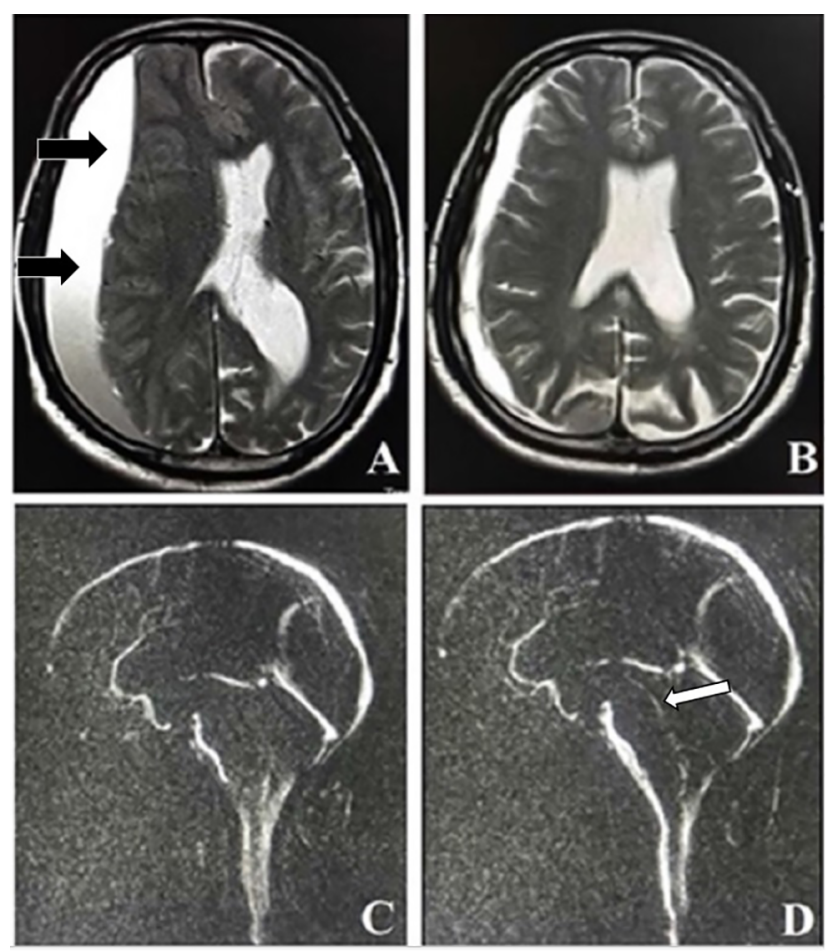

Fig. 1 (A-D) A 78-year-old male with subdural hematoma. Axial T2-W images show large subdural hematoma preoperatively (A) (black arrows) and postoperatively (B). Qualitative magnitude images show increase in CSF flow postoperatively across aqueduct (C and D) (white arrow). CSF, cerebrospinal fluid; T2-W, T2-weighted.

Table 3 Correlation of postoperative change in peak CSF velocity with changes in clinical outcome

\begin{tabular}{|c|c|c|c|c|c|}
\hline $\begin{array}{l}\text { Patient } \\
\text { group }\end{array}$ & Clinical outcome & $n$ & $\begin{array}{l}\text { Mean change in peak CSF velocity } \\
(\mathrm{cm} / \mathrm{s})\end{array}$ & SD & $p$ \\
\hline \multirow[t]{2}{*}{ Group I } & Improved & 2 & 2.24 & 0.08 & $\begin{array}{l}0.040^{\mathrm{a}} \text { (improved vs. } \\
\text { others) }\end{array}$ \\
\hline & Others & 2 & 0.55 & 0.01 & \\
\hline \multirow[t]{2}{*}{ Group II } & Improved & 21 & 0.28 & 0.49 & $\begin{array}{l}0.040^{\mathrm{a}} \text { (improved vs. } \\
\text { others) }\end{array}$ \\
\hline & Others & 16 & 0.02 & 0.10 & \\
\hline
\end{tabular}

Abbreviations: CSF, cerebrospinal fluid; SD, standard deviation. aSignificant. 
in - Table 3. In addition, patients with clinical improvement showed a higher percentage increase in peak CSF velocity (28.9\%) versus those who demonstrated worsening or no change (7\%). - Fig. 1 illustrates reduced flow across cerebral aqueduct in a 78-year-old male with subdural hematoma showing improvement in flow in cine MR across cerebral aqueduct postoperatively and corresponding improvement clinical symptoms. In addition, the changes in CSF flow dynamics in a 25-year-old female with cerebellar tonsillar herniation and cervical syrinx is shown in - Fig. 2.

Furthermore, an increase was seen in area of SAS postoperatively at ROI (mean area of SAS preoperatively: $1.10 \pm$ $1.13 \mathrm{~cm}^{2}$ and postoperatively: $1.19 \pm 1.18 \mathrm{~cm}^{2}$ ) which was found to be statistically significant $(p=0.03)$. The postoperative increase in area of SAS in a 64-year-old female with a dorsal intraspinal extramedullary lesion with resultant normalization of flow and increase in CSF peak velocity (preoperatively, $-2.76 \mathrm{~cm} / \mathrm{s}$ and postoperatively, $-3.57 \mathrm{~cm} / \mathrm{s}$ ) and increase in area of SAS $\left(\sim-0.64 \mathrm{~cm}^{2}\right)$ has been shown in -Fig. 3. Similarly, postoperative increase in area of SAS in a 44-year-old male patient with degenerative disc bulge and corresponding improvement in CSF flow dynamics is shown in - Fig. 4.

\section{Discussion}

The increasingly widespread capabilities and availability of MR imaging over the past decade have allowed better assessment of craniospinal disorders. PCMRI allows noninvasive evaluation of quantitative biomarkers, as well as dynamic information, pertaining to CSF flow alterations allowing postsurgical assessment. ${ }^{5}$ To the best of our knowledge, assessment of CSF flow dynamics at multiple craniospinal levels and role of temporal assessment in such patients is as yet unevaluated, wherein lies the strength of our methodology.

Increased emphasis has been placed on evaluation of CSF peak velocities in healthy volunteers; however, the reference standards at spinal levels are lacking. ${ }^{6}$ In this study, in control patients, the CSF velocities in the SAS across various levels were relatively uniform and did not present with any relevant differences. Similar parameters were reported in a study done by Wang et al inclusive of intraspinal CSF dynamics with measurements at foramen magnum, C7, and T7 levels. ${ }^{7}$ The small differences in CSF flow parameters at various levels are multivariate and can be attributed to anatomy of the CSF spaces, blood pressure, heart rate, compliance of surrounding tissues, and possible systematic errors like selecting the ROI

\section{Preoperative}
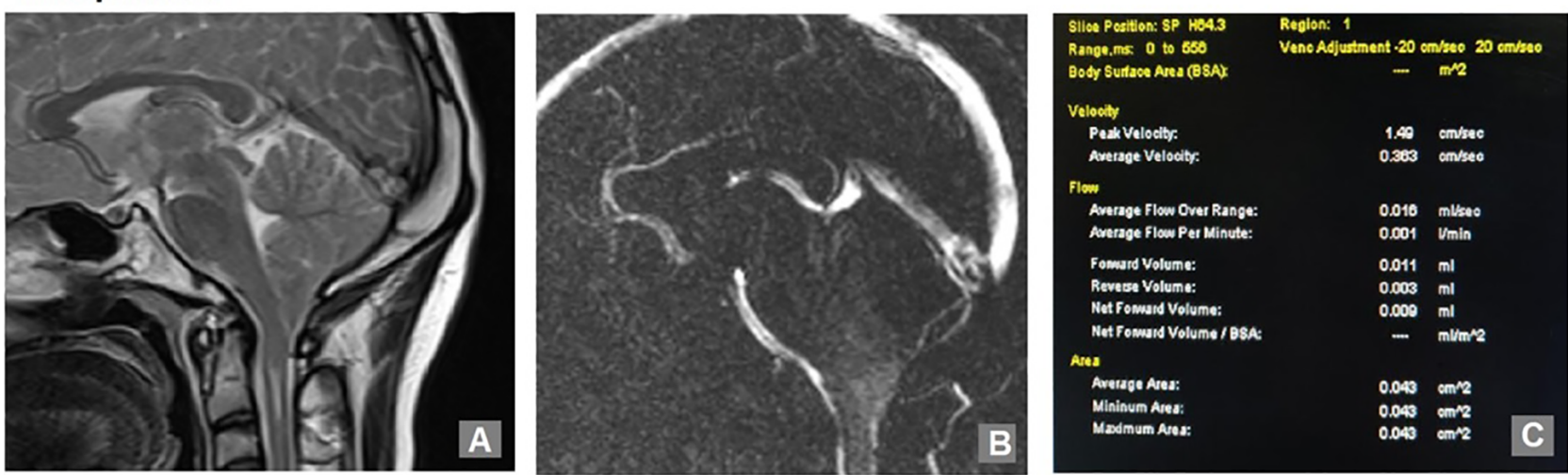

\section{Postoperative}
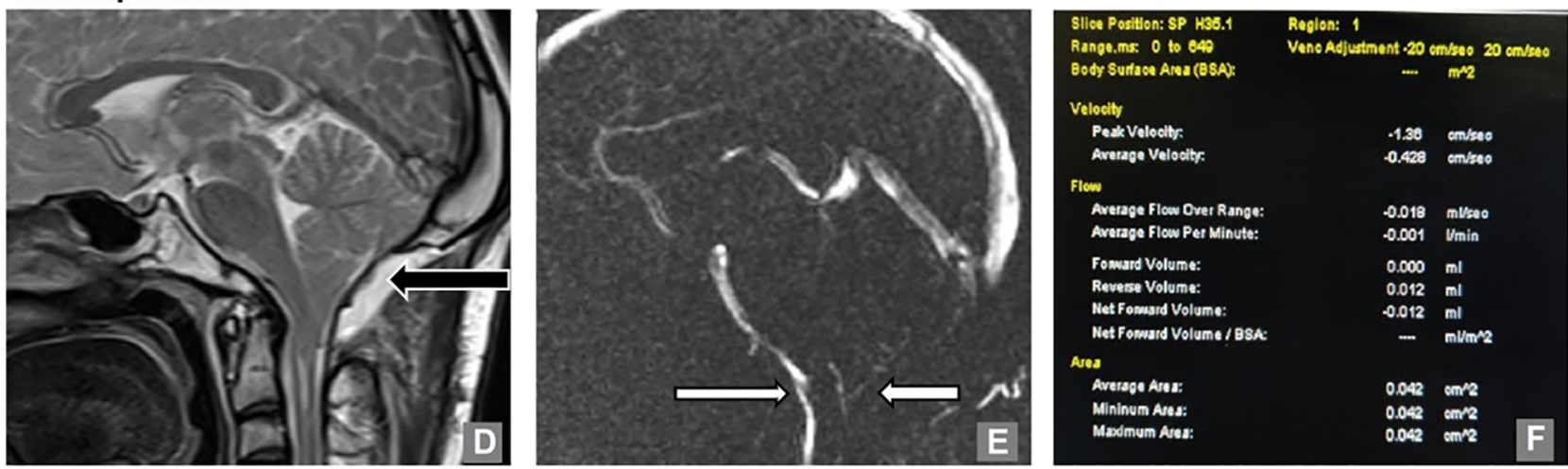

Fig. 2 (A-F) A 25-year-old female with cerebellar tonsillar herniation and cervical syrinx. Sagittal T2-WI (A) shows cerebellar tonsillar herniation and cervical syrinx with narrowing at craniovertebral junction and reduced CSF flow on qualitative magnitude images (B). Preoperative quantitative flow parameters (C). Postsurgical posterior fossa decompression (black arrow) on sagittal T2-WI (D) with improvement in qualitative flow (E) at the level of craniovertebral junction (F) Reduction in peak CSF velocity. CSF, cerebrospinal fluid; T2-WI, T2-weighted image. 
which can be subject to interobserver variations. To reduce interobservers' variability, the averages of mean quantitative values were taken and in case of significant inconsistencies,
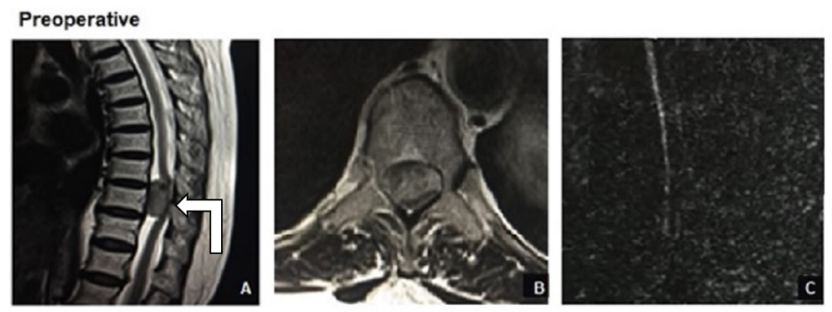

Postoperative
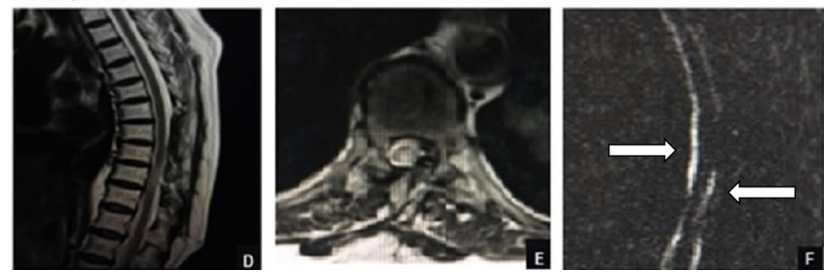

Fig. 3 (A-F) A 64-year-old female with a dorsal intraspinal extramedullary lesion. Intraspinal extramedullary lesion in dorsal region preoperatively (elbow arrow) in T2-WI sagittal (A) and axial (B). Qualitative magnitude images showing reduced flow ventrally and dorsally (C). Postoperative resolution of lesion (D) with increase in SAS (E) and improvement in flow on magnitude images (F) (arrows). SAS, subarachnoid spaces; T2-WI, T2-weighted image. the values were confirmed by another investigator. Further peak CSF velocity was used for comparisons among the different flow parameters, as it has shown less interobserver variations and less sensitive to systematic errors as mentioned in previous studies. ${ }^{8}$

The changes of metrics in at ROI in site of pathology corroborate favorably with alteration of parameters reported by previous investigators. ${ }^{6}$ Twenty-seven of our patients had intracranial pathology, while 23 had intraspinal pathology. Intracranial flow alteration in our study included patients with intracranial/intraventricular masses altering CSF flow. We observed reduced/absent flow on cine MR images with reduced CSF quantitative parameters and irregular low-amplitude peak velocity waveforms in both groups. Postoperatively, there was increase in pulsatile CSF flow with increase in quantitative values. Mass lesions, whether infratentorial or supratentorial, both indirectly caused aqueductal obstruction by virtue of compression of the posterior third ventricle or cerebral aqueduct. The degree of CSF flow compression showed relative diminution of flow on cine MR images. Increased pulsatile flow was noted from the inferior portion of the fourth ventricle into the cisterna magna suggesting flow redistribution. This was consistent with study by Quencer et al which showed similar changes allowing assessment of level of obstruction based on changes in flow patterns. ${ }^{9}$

\section{Preoperative}
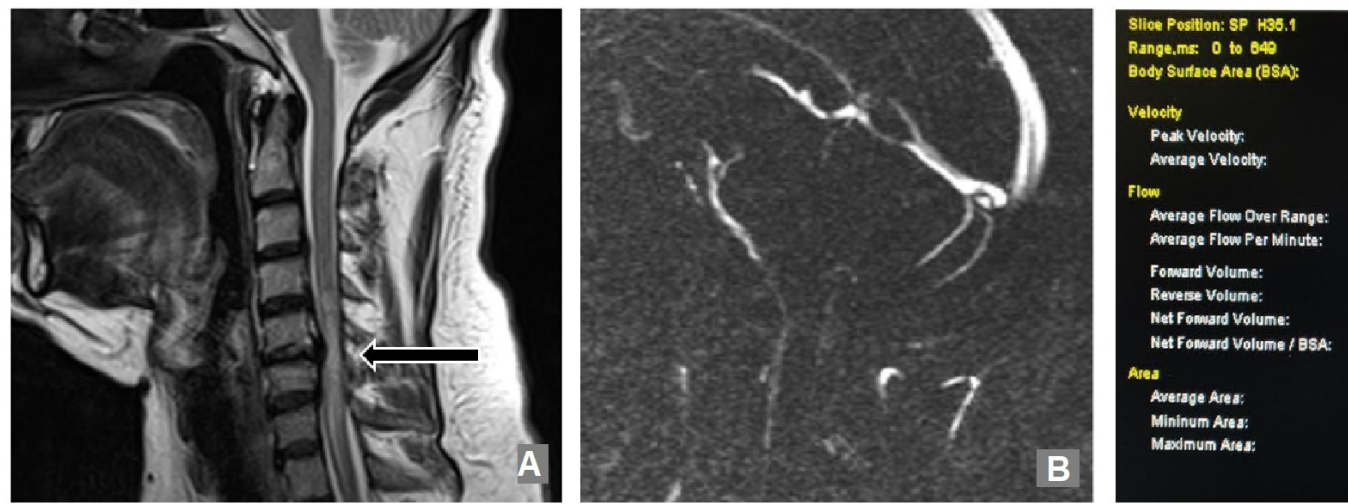

Region: 1 Vene Adjustment .20 embeo 20 ombeo -.. $\mathrm{m}^{\mathrm{N} 2}$ $-1,38$ intsec -0.428 mintase $-0.018 \mathrm{~m} / \mathrm{sec}$ -0.001 VImin $0.000 \mathrm{ml}$ $0.012 \mathrm{ml}$ $-0.012 \mathrm{ml}$ -.. mimns $0.042 \quad 6 m^{2} 2$ $0.042 \mathrm{~cm} / 2$ C

\section{Postoperative}
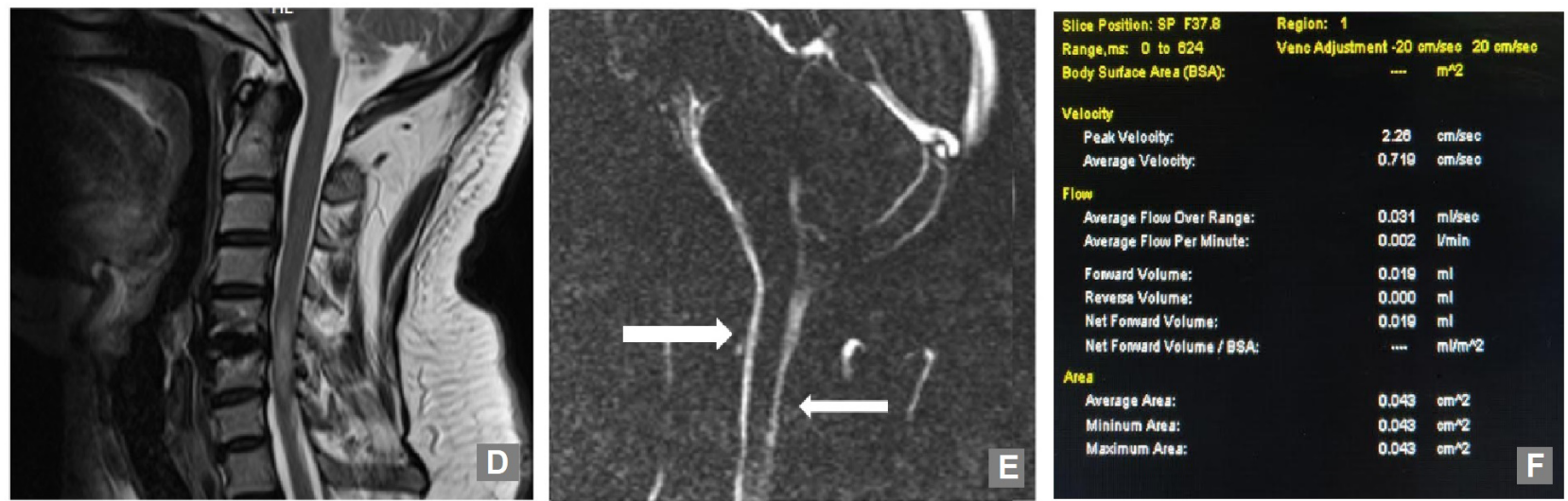

Fig. 4 (A-F) A 44-year-old male with degenerative disc bulge. (A) Degenerative disc bulge at C5-6 intervertebral level (black arrow) on sagittal T2-WI causing spinal canal narrowing and cervical cord compression with reduced flow on qualitative magnitude images (B). Reduced quantitative flow parameters. (C) Postoperative reduction in disc bulge (D) and improvement in CSF flow on qualitative magnitude images (E) and increase in peak CSF velocity (F). CSF, cerebrospinal fluid; T2-WI, T2-weighted image. 
Different pathogenetic mechanisms have been proposed causing varying alteration of CSF flow at craniocervical junction and rest of the SAS. It was suggested by Bhadelia et al and Oldfield et al that the rapid caudal tonsillar movements in Chiari's malformation act as a piston leading to increased systolic velocities and in contrast elsewhere a more obstructive nature of flow is seen leading to reduction in flow velocity. ${ }^{10,11}$ Postoperatively, in our study following surgical intervention, a different response in quantitative biomarkers was seen in group-I patients. The complex CSF flow dynamics at the foramen magnum, the anterior, and posterior cervical spinal canal in patients with tonsillar herniation plausibly alters the dynamics in a different manner, probably due to abnormal brainstem motion and tonsillar piston pressure effect. Adhesions obstructing or redirecting flow may also alter CSF dynamics in addition to tonsillar descent. The discrepancies in postoperative changes in quantitative values across the levels might be attributed to the regional variability in CSF dynamics instead of degree of CSF flow alteration. ${ }^{12,13}$

Intraspinal flow alterations in our study comprised those with flow alteration sequelae to injury/collection or mass lesion. In all such cases, we observed pattern of CSF flow alteration similar to intracranial pathologies both qualitatively and quantitatively at ROI with postoperative elevation in CSF peak velocities. The narrowing or obliteration of flow at spinal SAS leads to a nonlaminar/absent CSF flow with redistribution of pressure dynamics. In such patients, surgical decompression increases the spinal canal volume and causes widening of SAS with resultant augmentation of flow at the region of compression with regularization of pulsatality of temporal CSF velocity waveform. Qualitative dynamic cine images demonstrated a return to near-normal CSF dynamics on follow-up in most of the patients, reflecting the predicted mechanical response of CSF flow pulsations to decompressive surgery.

\section{Clinical Symptomatology and Flow Dynamics}

Postoperatively, there was a significant correlation in improvement in clinical outcome with change in CSF velocity in groups I and II, increasing with degree of mean change in CSF velocity. Improvement of disability score was seen in these patients. An improved CSF velocity profile following surgery in such patients may be a useful guide to anticipate a symptomatic improvement. Given the multifactorial etiology of symptoms in our patients, the improvement in surgical response observed in patients may be due to greater alteration of CSF flow as a more direct result of anatomical flow disturbance. In addition, the preoperative symptomatology may vary as per the chronicity of the lesion most commonly due to development of fibroglial adhesions leading to difference in outcome.

Further, a majority of the patients with improvement in symptoms showed a combined ventral and dorsal CSF flow abnormality on preoperative MRI. The higher improvement in patients with both ventral and dorsal flow alterations is possibly due to volumetric displacement of flow in singular flow (i.e., only either ventral or dorsal flow) alteration.
Findings were consistent with a previous study done by du Boulay. ${ }^{14}$ This may have implication in selection of patients that are preferred candidates for surgical decompression and are more likely to have a better clinical end result.

Our study had few limitations. Manual segmentation technique is prone to technical errors resulting in inaccurate estimation of CSF flow parameters. Quantitative values are underrepresented in regions of complex flow alterations. Further, spinal region is subject to pulsation artifacts and a higher signal-to-noise ratio altering value parameters.

\section{Conclusion}

Cine MR images provide dynamic information about level and degree of CSF flow obstruction and may be used in conjunction with conventional MR sequences for better evaluation of craniospinal flow alterations. Regional variability in CSF dynamics is seen at craniocervical junction (CCJ) and rest of SAS with distinct change in quantitative flow values following surgical correction. Improvement in CSF flow postsurgically correspondingly reflects in clinical outcome. However, future studies inclusive of a long-term follow-up and with technical improvements in PCMR technique will help further investigate our results. Further, a larger sample size and separate evaluation of CCJ and rest of spine may improve our understanding of flow dynamics in such cases.

\section{Declaration of Patient Consent}

The authors certify that they have obtained all appropriate patient consent forms. In the form, the patient(s) has/have given his/her/their consent for his/her/their images and other clinical information to be reported in the journal. The patients understand that their names and initials will not be published and due efforts will be made to conceal their identity, but anonymity cannot be guaranteed.

\section{Financial Support and Sponsorship \\ None.}

\section{Conflicts of Interest}

There are no conflicts of interest.

\section{References}

1 Bradley WG Jr. CSF Flow in the brain in the context of normal pressure hydrocephalus. AJNR Am J Neuroradiol 2015;36(5):831-838

2 Feinberg DA, Mark AS. Human brain motion and cerebrospinal fluid circulation demonstrated with MR velocity imaging. Radiology 1987;163(3):793-799

3 Negahdar MJ, Shakeri M, McDowell E, et al. Cine phase-contrast MRI measurement of CSF flow in the cervical spine: a pilot study in patients with spinal cord injury. In: Weaver JB, Molthen RC, eds. Proceedings of SPIE: Medical Imaging 2011: Biomedical Applications in Molecular, Structural, and Functional Imaging. SPIE:2011

4 McGirt MJ, Atiba A, Attenello FJ, et al. Correlation of hindbrain CSF flow and outcome after surgical decompression for Chiari I malformation. Childs Nerv Syst 2008;24(7):833-840

5 Battal B, Kocaoglu M, Bulakbasi N. Husmen G, Tuba Sanal $\mathrm{H}$, Tayfun C. Cerebrospinal fluid flow imaging by using 
phase-contrast MR technique. Br J Radiol 2011;84(1004): 758-765

6 Nitz WR, Bradley WG Jr, Watanabe AS, et al. Flow dynamics of cerebrospinal fluid: assessment with phase-contrast velocity MR imaging performed with retrospective cardiac gating. Radiology 1992;183(2):395-405

7 Wang Y, Xie J, Zhao Z, Zhang Y, Li T, Si Y. Changes in CSF flow after one-stage posterior vertebral column resection in scoliosis patients with syringomyelia and Chiari malformation type I. J Neurosurg Spine 2013;18(5):456-464

8 Korbecki A, Zimny A, Podgórski P, Sąsiadek M, Bladowska J. Imaging of cerebrospinal fluid flow: fundamentals, techniques, and clinical applications of phase-contrast magnetic resonance imaging. Pol J Radiol 2019;84:e240-e250

9 Quencer RM, Post MJ, Hinks RS. Cine MR in the evaluation of normal and abnormal CSF flow: intracranial and intraspinal studies. Neuroradiology 1990;32(5):371-391

10 Bhadelia RA, Bogdan AR, Wolpert SM, Lev S, Appignani BA, Heilman CB. Cerebrospinal fluid flow waveforms: analysis in patients with Chiari I malformation by means of gated phase-contrast MR imaging velocity measurements. Radiology 1995;196(1):195-202

11 Oldfield EH, Muraszko K, Shawker TH, Patronas NJ. Pathophysiology of syringomyelia associated with Chiari I malformation of the cerebellar tonsils. Implications for diagnosis and treatment. J Neurosurg 1994;80(1):3-15

12 Dolar MT, Haughton VM, Iskandar BJ, Quigley M. Effect of craniocervical decompression on peak CSF velocities in symptomatic patients with Chiari I malformation. AJNR Am J Neuroradiol 2004;25(1):142-145

13 Iskandar BJ, Quigley M, Haughton VM. Foramen magnum cerebrospinal fluid flow characteristics in children with Chiari I malformation before and after craniocervical decompression. J Neurosurg 2004;101(2, suppl):169-178

14 Du Boulay GH. Pulsatile movements in the CSF pathways. $\mathrm{Br}$ J Radiol 1966;39(460):255-262 